Rel at i onshi ps bet ween st r et ch- short eni ng cycle perf or mance and maxi mum miscl e st rengt $h$

\begin{tabular}{|l|l|}
\hline 著者 & M yaguchi Kazuyoshi, Demur a Shi ni chi \\
\hline $\begin{array}{l}\text { j our nal or } \\
\text { publ i cat i on ti t l e }\end{array}$ & Journal of Strengt h and Condi t i oni ng Research \\
\hline vol une & 22 \\
\hline number & 1 \\
\hline page range & $19-24$ \\
\hline year & 2008 01-01 \\
\hline URL & ht t p: //hdl . handl e. net /2297/19132 \\
\hline
\end{tabular}

doi: https://doi.org/10.1519/jsc.0b013e31815f2f94 


\title{
ReLATIONSHIPS BETWEEN STRETCH-SHORTENING Cycle Performance and Maximum Muscle Strength
}

\author{
Kazuyoshi Miyaguchi ${ }^{1}$ and Shinichi Demura ${ }^{2}$ \\ ${ }^{1}$ Liberal Arts Education Center, Ishikawa Prefectural University, Ishikawa, Japan; ${ }^{2}$ Faculty of Education, Kanazazwa \\ University, Kakuma, Japan
}

\begin{abstract}
This study aimed to examine the relationships between muscle power output using the stretch-shortening cycle (SSC) and maximum strength, as measured by the 1 RM (1 repetition maximum) test and the isokinetic dynamometer under elbow flexion. Sixteen trained, young adult males pulled a constant load of $40 \%$ MVC (maximum voluntary elbow flexion contraction) by ballistic elbow flexion under the following two preliminary conditions: 1) the static relaxed muscle state (SR condition) and 2) using the SSC (SSC condition). Muscle power was determined from the product of the pulling velocity and load mass by a power measurement instrument with a rotary encoder. The $1 \mathrm{RM}$ bench press (1RM BP) and isokinetic maximum strength under elbow flexion with the Cybex-325 were measured as indicators of dynamic maximum strength. 1) The early power output exerted under the SSC condition showed a significant and high correlation with the $1 \mathrm{RM}$ BP $(r=0.83)$, but only moderate correlation with the isokinetic muscle strength $(r=0.50-0.67)$. 2) The contribution of the 1 RM BP to the early muscle contraction velocity exerted under the SSC condition was large. These results suggested that muscle power exerted using the SSC shows a stronger relationship with maximum muscle strength measured by a $1 \mathrm{RM}$ test rather than isokinetic maximum strength.
\end{abstract}

KEY WoRDS muscle power, elbow flexion, 1 RM bench press, isokinetic strength, counter-movement

\section{INTRODUCTION}

I n competitive sports that require explosive power output from the upper limbs, such as "throwing" or "hitting," a counter-movement (e.g., back-swing) using a Stretch-Shortening Cycle (SSC) of concentric muscle

Address correspondence to Kazuyoshi Miyaguchi, kazu1060@lapis. plala.or.jp.

$1533-4287 / 22(1) / 19-24$

Journal of Strength and Conditioning Research

(C) 2008, National Strength and Conditioning Association action following eccentric muscle action is frequently used (22). The muscle power output exerted from concentric contraction using the SSC produces greater power output in a shorter period of time than that from a simple concentric contraction only $(5,17,27)$. Athletes perform various types of strength training to improve the ability of their muscles to exert power using the SSC.

An exercise using SSC is performed at high speed by dynamic muscle contraction. Recently, to improve muscle strength, isokinetic training to control the exercise velocity by using an isokinetic machine (Cybex) has been performed. This machine matches the resistive force to the muscle force in order to maintain constant joint angular velocity. This machine is very expensive, and it has also been pointed out that measurements differ depending on the type of machine (28). In addition, many athletes cannot use it at the same time.

As a result, many athletes perform weight training for muscle strength improvement. In particular, the bench press is a typical exercise for the upper body, and its one repetition maximum (1 RM) is the index of muscular power development (24). In the bench press, lifters must exert muscle strength reactively and ballistically to overcome the inertia of the weight, such as a barbell, early in the lift. Although the strength exerted by the bench press is the same dynamic strength, it is considerably different from the above-stated isokinetic strength in a muscle force exertion pattern. Also, the bench press includes a change from extension to contraction in force exertion, and it is thought to have a high correlation to muscle power output using the SCC (SSC performance) rather than the strengths exerted by isokinetic muscle contraction. Therefore, it is often used by power athletes aiming to improve performance, although the motion of the bench press is different from the actual movement such as throwing or smashing.

However, the relationship between performance produced using the SSC and the above-stated dynamic strength is unclear. In addition, few studies have examined relationships between the maximum rate of rise and the dynamic strength. As a result, the bench press is frequently performed by athletes, although the purpose of the bench press, whether for 
improvement of strength or muscle contraction velocity, is unclear. Therefore, the hypothesis that, "muscle power exerted using the SSC shows a higher correlation with maximum muscle strength measured by a $1 \mathrm{RM}$ test rather than isokinetic maximum strength" was proposed in this study, and we attempted to test it.

This study aimed to examine relationships between muscle power output using the SSC and maximum strengths measured by the $1 \mathrm{RM}$ test and isokinetic dynamometer in elbow flexion.

\section{Methods}

\section{Approach to the Problem}

Until now, studies on SSC have been performed using mainly jumping $(1,4,25)$. However, this movement comprises an entire body movement and performance is considerably affected by technical factors (e.g., forward and upward arm swings), and quantification of muscle power is difficult because of the use of body weight as a load (9). Hence, the elbow flexion was selected in this study. This motion is not affected by body weight, can isolate the muscle groups related to the movement, and can also utilize SSC with counter-movement easily. Hence, it was judged to be valid as a measurement movement.

In addition, to examine relationships between muscle power output using the SSC and the maximum strength measured by the $1 \mathrm{RM}$ test and isokinetic dynamometer, the static preliminary relaxed arm muscle state (SR condition) without using the SSC was selected as a control condition.

\section{Subjects}

The subjects consisted of sixteen trained, young adult males (mean age $21.4 \pm 0.9$ years, height $1.74 \pm 0.05 \mathrm{~m}$, and body mass $71.0 \pm 7.9 \mathrm{~kg}$ ). They were selected from the following sports backgrounds: baseball [5], basketball [2], swimming [3], track and field [5], and soccer [1]. Their mean training age was $12.1 \pm 1.5 \mathrm{yr}$. Their prior weight training experiences ranged from $0.5-8$ years. The power test was performed with the dominant arm, determined by Oldfield's (23) handedness inventory because of the tendency towards dominant hand use in throwing or hitting. Informed consent was obtained from all subjects after a full explanation of the experimental project and its procedures. The study was approved by the Human Rights Committee of Kanazawa University.

\section{Procedures}

Experimental Device and Muscle Power Measurement. Muscle power was measured using a muscle power measurement instrument that adopted a weight loading method developed by Ikemoto et al. (12) (Yagami, Japan) (Figure 1). This measurement device consists of a rotary encoder (SUNX, ORE38-1200) attached to a fixed pulley and a recording device. The rotary encoder measures the rotational angle with a sampling frequency of $100 \mathrm{~Hz}$ via an analog-to-digital interface. The rotational angle was converted to the pulling velocity of the wire rope with the load in the recording device. The muscle power was drawn from the product of the pulling velocity and load mass based on Newton's second law of motion.

The subjects sat in an adjustable ergometric chair sideways, and put their right-arm on a table. They then put their axilla on the edge of the table with supination of the forearm. A bowling protector was worn to restrict the movement of the wrist. Subjects touched their palm to the handle, and explosively pulled the handle by elbow flexion as quickly

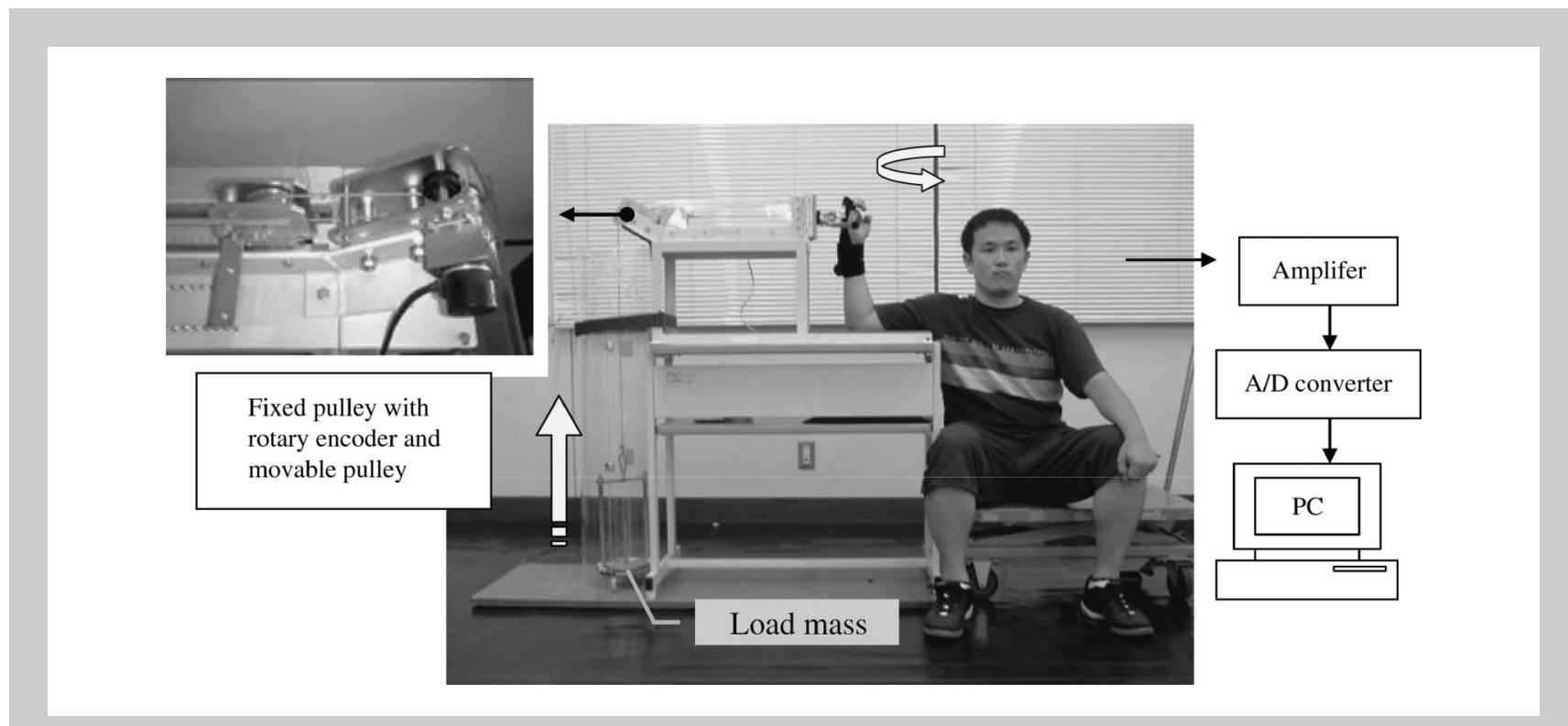

Figure 1. Schematic representation of experimental set up. 
as possible in the opposite direction of a wire rope that was connected to a constant load mass. The range of motion of the elbow flexion was from $80^{\circ}$ to $120^{\circ}$ (full-extension angle was $0^{\circ}$ ), and the starting position angle was $80^{\circ}$. To account for systematic error, the rotary encoder and load cell were calibrated before each measurement. Test-retest reliability was at peak power ICC $=0.89$, and the cross correlation coefficients between trials for time-series power parameters exerted from each preliminary condition were high $(\mathrm{r}=0.75$ $0.99, P<0.05)(20)$.

To determine submaximal loads for the power test, the subjects performed the maximal voluntary elbow flexion test twice in succession, and the higher value was chosen as the MVC. This test was carried out by maximal isometric contraction with the same angle $\left(80^{\circ}\right)$ as starting position angle. The MVC was measured by a grip dynamometer with a spring scale attached to the end of the wire. In previous studies $(2,14,21)$, it was reported that the maximum power was exerted at $30-45 \%$ MVC. Therefore, the load was set at $40 \%$ MVC in this study.

Experimental Conditions. The two preliminary conditions were as follows.

1) SR condition: Each subject pulled the handle from a static relaxed arm muscle state, keeping an $80^{\circ}$ elbow joint angle by concentric contraction.

2) SSC condition: Each subject pulled the handle with the same load using a voluntary countermovement according to the subject's original rhythm and timing within the range of $80^{\circ}-120^{\circ}$. Because subject's best SSC performance could not be demonstrated when the restriction of the counter-movement was severe, the frequencies of the countermovement were assumed to be arbitrary. The starting angle of the concentric contraction on elbow flexion was determined by a beeping sound from a device at a position of 80 degrees, and several rehearsals were performed by the subject.

The power test was performed twice for the abovestated two conditions, and the higher value was used as the analysis data.

Measurement of Maximum Strength on Various Muscle Actions. 1) $1 \mathrm{RM}$ bench press (1 RM BP)

$1 \mathrm{RM} \mathrm{BP}$ within one month was used as an indicator of a 1 RM test.

2) Isokinetic maximum strength (Isokinetic strength).

An isokinetic dynamometer (Cybex-325, Lumex, USA) was used to measure isokinetic maximum strength. We measured peak torque in elbow flexion at three angular velocities, $60^{\circ} \cdot \mathrm{sec}^{-1}$ (three trials), $180^{\circ} \cdot \mathrm{sec}^{-1}$ (four trials), and $300^{\circ} \cdot \mathrm{sec}^{-1}$ (five trials) referring to the protocol of previous work (15). The highest value was adopted as a peak torque for each measurement condition.

\section{Evaluation Parameters}

The use of SSC in the upper limbs is extremely effective in enhancement of initial muscle contraction velocity (20). Therefore, the following muscle power parameters were selected in reference to a previous study (20): 1) $0.1 \mathrm{~s}$ velocity during concentric contraction $(\mathrm{m} / \mathrm{s}), 2)$ peak velocity $(\mathrm{m} / \mathrm{s})$, 3) time to peak velocity (s), 4) $0.1 \mathrm{~s}$ initial power (W), 5) peak power (W) (Figure 2).

Peak power was based on the following equation:

Peak power $(\mathrm{W})=$ load $(\mathrm{kg}) \times$ gravitational acceleration

$$
\left(9.80665 \mathrm{~m} / \mathrm{s}^{2}\right) \times \text { peak velocity }(\mathrm{m} / \mathrm{s})
$$

\section{Statistical Analyses}

The paired $t$ test was used to reveal mean differences between two conditions for muscle power parameters. The effect size (ES) was calculated to examine the size of the mean differences. The relationship between muscle power parameters and various maximum muscular strengths were examined using Pearson's correlation coefficient. The criterion level for significance was set at $P \leq 0.05$. The level of statistical significance was based on Bonferroni's method. This method is distributed equally in 0.05 pair comparisons, by dividing 0.05 by the total of pair comparisons.

\section{Results}

Table 1 shows the results of muscle strength measurements. Table 2 shows parameters exerted from the SR and SSC conditions and the test results of mean differences. There were no significant differences between SSC and SR conditions for the peak velocity and peak power. However, the time to peak velocity from the SSC condition was significantly shorter than that of the SR condition. As for $0.1 \mathrm{~s}$ power, the SSC condition $(36.1 \mathrm{w})$ was twice as large as the SR condition $(15.0 \mathrm{w})$, and the ES of the $0.1 \mathrm{~s}$ velocity was very large at 3.14 .

Figure 3 shows the relationships of $0.1 \mathrm{~s}$ velocity between SR and SSC conditions, and also the relationships of peak velocity between both conditions. Peak velocities in the SR

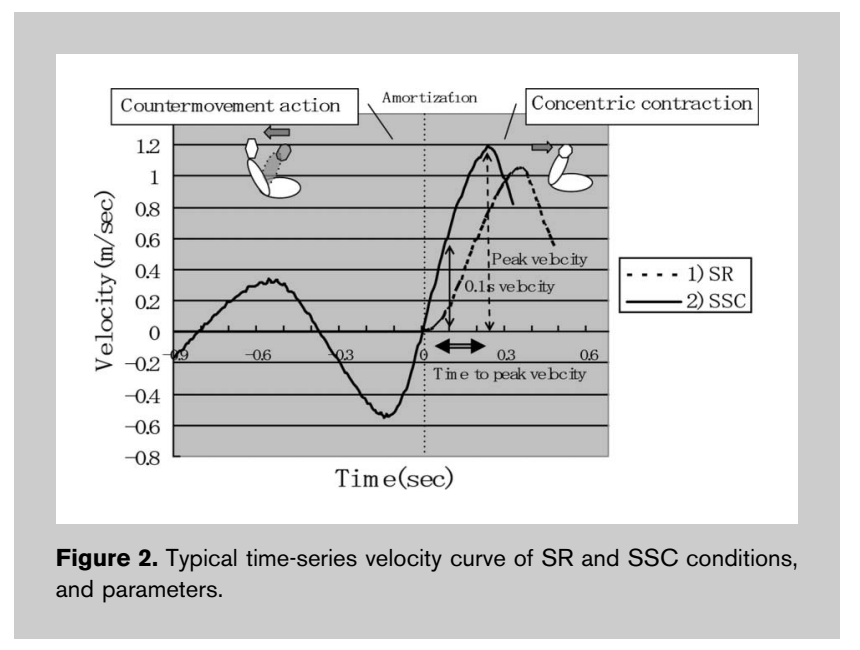


TABLE 1. Physical characteristics of the subjects and results of maximum strength test.

\begin{tabular}{lcccccccc}
\hline & & & & & \multicolumn{4}{c}{ Isokinetic FLEXION (ft lbs) } \\
\cline { 6 - 8 } & Age $(\mathrm{yrs})$ & Height $(\mathrm{m})$ & Weight $(\mathrm{kg})$ & MVC $(\mathrm{N})$ & $60 \mathrm{deg} / \mathrm{s}$ & $180 \mathrm{deg} / \mathrm{s}$ & $300 \mathrm{deg} / \mathrm{s}$ & $1 \mathrm{RM}$ BP $(\mathrm{kg})$ \\
\hline Mean & 21.40 & 1.74 & 70.50 & 98.43 & 24.63 & 22.88 & 19.69 & 85.47 \\
SD & 0.90 & 0.05 & 7.87 & 20.69 & 3.93 & 3.36 & 3.75 & 15.90 \\
Range & $18-23$ & $1.61-1.81$ & $61-86$ & $73.53-137.25$ & $19-33$ & $17-29$ & $15-27$ & $60-115$ \\
\hline
\end{tabular}

condition showed a significant and high correlation $(\mathrm{r}=0.72)$ with those in the SSC condition, but $0.1 \mathrm{~s}$ velocities showed an insignificant correlation.

Table 3 shows correlations between $0.1 \mathrm{~s}$ velocity and $0.1 \mathrm{~s}$ power and various muscular strength values in both conditions. Although significant correlations were not found in the SR condition, the $0.1 \mathrm{~s}$ power in the SSC condition showed a significant and high correlation with the 1 RM BP $(\mathrm{r}=0.83)$, and moderate correlation with the isokinetic strength $(\mathrm{r}=0.50-0.67)$. The $0.1 \mathrm{~s}$ velocity showed a significant correlation only with the $1 \mathrm{RM} \mathrm{BP}(\mathrm{r}=0.62)$.

\section{Discussion}

The ability to exert power using the SSC differs considerably for each individual $(25,27)$, and the elastic properties of the tendons and muscle-tendon complex (MTC) produce peculiar changes according to training loads and muscle contraction types $(18,19)$. It is possible that the muscle strength that was improved by training affects SSC performances.

As for peak velocity and peak power, there was no significant difference between the SR and SSC condition. This result was different from previous reports $(4-7,17)$ that examined the use of the lower limbs. As an example, Komi et al. (17) reported that jumping height and power output produced by a drop jump with counter-movement are larger than those of the squat jump without counter-movement. In the upper limbs, the SSC potentiation that is observed in the lower limbs may not appear conspicuously. Regarding this point, Glasheen et al. (10) pointed out that the upper limbs do not have tendon tissues such as the Achilles tendon which have strong elastic properties.

On the other hand, $0.1 \mathrm{~s}$ velocity and $0.1 \mathrm{~s}$ power exerted from the SSC condition showed higher values than those from the SR condition, and an increase in the early power output was marked. Because the difference between both conditions in $0.1 \mathrm{~s}$ velocity was very large, it is considered that using the SSC increased the initial muscle contraction velocity ( $0.1 \mathrm{~s}$ velocity), and as a result, the early power output increased. Also, previous studies $(20,26)$ reported that the potentiation of SSC was marked in the first half of concentric contraction. Considering that the coupling time (ground contact time) of the drop jump, which is an indicator of ability to achieve SSC in lower limbs, is $0.1-0.2$ seconds (3), we should evaluate the SSC potentiation in a range of 0.1-0.2 sec when evaluating muscle functions related to throwing or hitting.

Peak velocities showed significant correlations between the SR condition and the SSC condition, but $0.1 \mathrm{~s}$ velocities did not between both conditions (Figure 3). Although physical abilities (physiological factors) related to peak velocity are similar to each other in the SR and SSC conditions, a different

TABLE 2. Significant differences between mean values of evaluation parameters for SSC and SR conditions.

\begin{tabular}{lcccrrr}
\hline & 1) SSC condition & 2) SR condition & & & \\
\cline { 2 - 3 } \multicolumn{1}{c}{ Parameters } & Mean $\pm S D$ & Mean $\pm S D$ & & $t$-value & & ES \\
\hline 0.1 s velocity (m/s) & $0.45 \pm 0.11$ & $0.19 \pm 0.04$ & & $9.89^{*}$ & $1)>2)$ & 3.14 \\
0.1 s power $(\mathrm{W})$ & $36.06 \pm 14.75$ & $14.98 \pm 4.85$ & & $7.09^{*}$ & $1)>2)$ & 1.92 \\
Peak velocity (m/s) & $1.17 \pm 0.11$ & $1.17 \pm 0.13$ & & 0.24 & n.s. & 0.00 \\
Peak power (W) & $91.94 \pm 20.48$ & $90.76 \pm 17.30$ & & 0.59 & n.s. & 0.06 \\
Time to peak velocity (s) & $0.32 \pm 0.05$ & $0.40 \pm 0.04$ & & $9.61^{*}$ & $1)<2)$ & -1.77 \\
\hline
\end{tabular}

Note: $\alpha^{\prime}=0.05 / 5=0.01, \mathrm{t}(15,0.01)=2.95 *$ : $t$-value $>2.95$.

$\mathrm{ES}=$ Effect size; n.s. $=$ Non significant. 


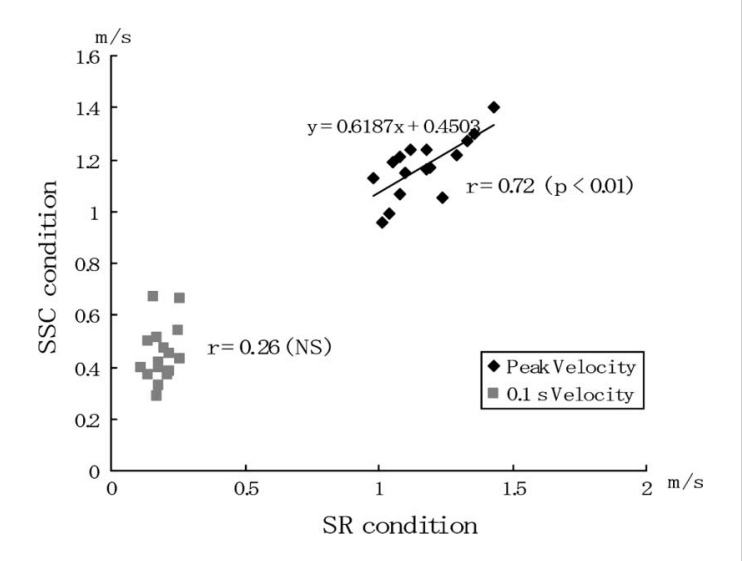

Figure 3. Relationships of peak and $0.1 \mathrm{~s}$ velocity between SR and SSC conditions.

factor may contribute to the $0.1 \mathrm{~s}$ velocity respectively. The biomechanical function of MTC is divided into the contractile component and the elastic component (11). It is inferred that the influence (recycling of elastic energy including stretch reflex) of the elastic component to passive force relates closely to initial muscle contraction velocity (0.1 s velocity) exerted from SSC condition. However, because the influence is temporary (initial phase) and a contractive force (active contractile component) of the muscles mainly contributes to a maximum velocity, a correlation of peak velocity between both conditions might have been high. As already discussed, a potentiation of SSC using this device has been observed only in the initial phase. Hence, we paid attention to relationships between early power output $(0.1 \mathrm{~s}$ velocity and $0.1 \mathrm{~s}$ power) and dynamic maximum strength measured by the isokinetic dynamometer and 1RM BP.

TABLE 3. Correlations between $0.1 \mathrm{~s}$ power and velocity from SR and SSC conditions and strength scores.

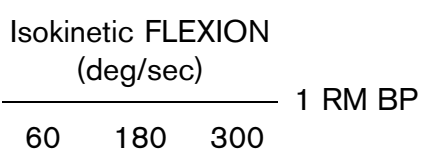

\begin{tabular}{lrrrr}
\hline SR condition & & & & \\
0.1 sec velocity & -.27 & -.04 & -.01 & -.18 \\
0.1 sec power & .21 & .40 & .48 & .41 \\
SSC condition & & & & \\
0.1 sec velocity & .26 & .44 & .45 & $.62^{\star}$ \\
0.1 sec power & $.50^{\star}$ & $.63^{\star}$ & $.67^{\star}$ & $.83^{\star}$
\end{tabular}

${ }^{*}$ Denotes significant correlations $(P<0.05)$.
It is important to note that the $0.1 \mathrm{~s}$ velocity showed a significant correlation with the $1 \mathrm{RM}$ BP only in the SSC condition. A functional adaptation of the nerve system related to specific power exertion in the $1 \mathrm{RM} \mathrm{BP}$ is considered to affect the early muscle contraction velocity in the SSC condition. Subjects with superior 1RM BP may send a large amount of nerve stimulus to the muscles intensively, and enable quick exertion of muscle power.

Elliott et al. (8) pointed out that the bench press is a very popular SSC movement that lends itself to the storage and release of strain energy. Thus, power exertion from elbow flexion using the SSC in this study may be very similar to that of the bench press. Actually, it is known that well-trained athletes can lift heavy barbells skillfully using a countermovement called "cheating" in the 1 RM BP test. Hence, the 1 RM BP might have shown a significant correlation with $0.1 \mathrm{~s}$ velocity in the $\mathrm{SSC}$ condition, even if the movement form or agonist muscle of elbow flexion of the measurement movement chosen by this study was different from those of the bench press. In contrast, the $1 \mathrm{RM}$ BP showed insignificant correlations with $0.1 \mathrm{~s}$ velocity in the SR condition. The above-stated results suggest that the $1 \mathrm{RM}$ BP especially contributes to muscle power exertion using the SSC.

In addition, when lifting a weight such as a barbell, high tension is developed in the muscles by isometric contraction to support the weight, even before the movement starts. This is called preloading. On the other hand, the isokinetic dynamometer with an accommodating-resistance apparatus does not load the muscle prior to the contraction. Although $1 \mathrm{RM}$ BP and isokinetic maximum strength involves the same dynamic muscular power exertion, the number of motor units recruited may differ when starting the movement. On the other hand, prestretching of SSC may be similar to preloading of bench press. Therefore, it is inferred that $0.1 \mathrm{~s}$ velocity in the SSC condition showed significant correlation with the 1 RM BP. In conclusion,

1) The early power output exerted from the SSC condition shows a significant and high correlation with the 1 RM BP $(r=0.83)$, but significant and moderate correlation with the isokinetic muscle strength $(\mathrm{r}=0.50-0.67)$.

2) The contribution of $1 \mathrm{RM}$ BP to early muscle contraction velocity under the SSC condition is large.

The hypothesis that, "muscle power exerted using the SSC shows a stronger relationship with maximum muscle strength measured by a $1 \mathrm{RM}$ test rather than isokinetic maximum strength" was supported from the above results.

\section{Practical Applications}

All competition sports involve acceleration (change in velocity per unit time) of the body and, for some sports, an implement (e.g., baseball, tennis racket) as well. Because of 
individual differences in the ability to exert force at different speeds (13), the maximum strength score obtained from isometric and low-speed lifting tests (1 RM test) may limit predicting performances in sports, such as tennis or baseball, that involve acceleration at high speed. Until now, the isokinetic muscle strength measurement has been frequently performed based on the definition of strength: the maximal force that a muscle group can generate at a specified velocity (16). However, the SSC is frequently used in these sports. Therefore, the maximum strength measured by a $1 \mathrm{RM}$ test is valid to evaluate the muscle function related to SSC performances, rather than isokinetic maximum strength.

\section{REFERENCES}

1. Asmussen, $\mathrm{E}$ and Bonde, PF, Storage of elastic energy in skeletal muscles in man. Acta Physiol Scand 91: 385-392, 1974.

2. Berger, R. Effect of dynamic and static training on vertical jumping. Research Quarterly 34: 419-424, 1963.

3. Bobbert, MF, Huijing, PA, and Van, GJ. Ingen Schenau. Drop jumping II: the influence of jumping technique on the biomechanics of jumping. Med Sci Sports Exerc 19: 332-338, 1987.

4. Bosco, C and Komi, PV. Potentiation of the mechanical behavior of the human skeletal muscle through prestretching. Acta Physiol Scand 106: 467-472, 1979.

5. Bosco, C, Komi, PV, and Ito, A. Prestretch potentiation of human skeletal muscles during ballistic movement. Acta Physiol Scand 111: 135-140, 1981.

6. Bosco, C, Tihanyi, J, Komi, PV, and Apor P. Store and recoil of elastic energy in slow and fast types of human skeletal muscle. Acta Physiol Scand 16: 343-349, 1982.

7. Bosco, C and Viitasalo, JT. Potentiation of myoelectric activity in human muscle in vertical jumps. Electromyo Clin Neuro 22: 549-562, 1982.

8. Elliott, BC, Wilson, GJ, and Kerr, G.K. A biomechanical analysis of the sticking region in the bench press. Med Sci Sports Exerc 21: $450-462,1989$.

9. Furuya, K, Funato, K, Takatoh, S, Mutoh, Y, and Miyashita, M. A new isokinetic dynamometer for measuring human leg extension power. J Sports Sci 5: 669-675, 1986.

10. Glasheen JW and McMahon, TA. Arms are different from legs: mechanics and energenics of human hand-running. J Appl Physiol 78: 1280-1287, 1995.

11. Hill, AV. The series elastic component of muscle. Proc R Soc Lond B Biol Sci 137: 273-280, 1950.

12. Ikemoto, Y, Demura, S, Yamaji, S, Nakada, M. Kitabayashi, T, and Nagasawa, Y. The characteristics of simple muscle power by gripping: gender differences and reliability of parameters using various loads. J Sports Med Phys Fitness 46: 62-70, 2006.

13. Jorgensen, K. Force-velocity relationship in human elbow flexors and extensors. In: Biomechanics V-A, PV Komi, ed. Baltimore: University Park Press. 1976.

14. Kaneko. M, Fuchimoto, H, Toji, H, and Suel, K. Training effect of different loads on the force-velocity relationship and mechanical power output in human muscle. Scand J Sports Sci 5: 50-55, 1983.

15. Kannus, P. Isokinetic evaluation of muscular performance: implications for muscle testing and rehabilitation. Int J Sports Med 15: 11-18, 1994.

16. Knuttgen, $\mathrm{H}$ and Kraemer, W. Terminology and measurement in exercise performance. J Appl Sport Sci Res 1: 1-10, 1987.

17. Komi, PV and Bosco, C. Utilization of stored lastic energy in leg extensor muscles by men and women. Med Sci Sports 10: 261-265, 1978.

18. Kubo, K, Kanehisa, H, Kawakami, Y, and Fukunaga, T. Elasticity of tendon structures of the lower limbs in sprinters. Acta Physiol Scand 168: 327-335, 2000a.

19. Kubo, K, Kanehisa, H, Kawakami, Y, and Fukunaga, Y. Elastic properties of muscle-tendon complex in long-distance runners. Eur. J Appl Physiol 81: 181-187, 2000b.

20. Miyaguchi, K and Demura, S. Muscle power output properties using the stretch-shortening cycle of the upper limb and their relationships with a one-repetition maximum bench press. J Physiol Anthrop 25: 239-245, 2006.

21. Moritani, T, Muro, M, Ishida, K, and Taguchi, S. Electrophysiological analyses of the effects of muscle power training. Res. J Phys Educ Jpn 1: 23-32, 1987.

22. Newton, RU, Murphy, AJ, Humphries, BJ, Wilson, GJ, Kraemer, WJ, and Häkkinen, $\mathrm{K}$. Influence of load and stretch shortening cycle on the kinematics, kinetics, and muscle activation that occurs during explosive upper-body movements. Eur J Appl Physiol 75: 333-342, 1997.

23. Oldfield, RC. The assessment and analysis of handedness: the Edinburgh inventory. Neuropsychologia 9: 97-113, 1971.

24. Thomas, RB and Roger, WE. Testing and Evaluation: Essentials of strength training and conditioning/National Strength and Conditioning Association 2nd ed. Human Kinetics, pp. 273-317, 2000.

25. Walshe, AD, Wilson, GJ, and Murphy, AJ. The validity and reliability of a test of lower body musculotendinous stiffness. Eur J Appl Physiol 73: 332-339, 1996.

26. Walshe, AD, Wilson, GJ, and GJC. Ettema Stretch-shorten cycle compared with isometric preload: contributions to enhanced muscular performance. J Appl Physiol 84: 97-106, 1998.

27. Wilson, GJ, Murphy, AJ, and Pryor, JF. Musculotendinous stiffness: its relationship to eccentric, isometric, and concentric performance. J Appl Physiol 76: 2714-2719, 1994.

28. Yamamoto, T. Measurement and Assessment for Practical and Scientific Conditioning. Book House HD. pp. 22-32, 2001. Tokyo. (in Japanese). 